\title{
A New Approach to the Design of a CNC Machine for Making Orthotic Moulds
}

\author{
Karlo OBROVAC, Pero RAOS, Tomislav GALETA, Josip NIŽETIĆ, Alan MUTKA, Jadranka VUKOVIĆ OBROVAC, Zoran VRHOVSKI
}

\begin{abstract}
This paper is about defining a different novel concept of a simple machine for producing spinal orthosis moulds. Should such a machine be developed, the required functionality with a special emphasis on low price is to be achieved. The paper considers the issues regarding the kinematics and feasibility confirmation as related to the concept. A CAD model made for the purpose of simulation and activities related to the software development for generating tool trajectories along with the belonging kinematics and trajectory visualization were carried out.
\end{abstract}

Keywords: CAD/CAM; O\&P; spinal orthosis; 3D scanning

\section{INTRODUCTION}

$\mathrm{CAD} / \mathrm{CAM}$ technologies have been used since the late 1950s. It was a period of intensive progress and increased demands from the automotive, aviation, military and space industry oriented towards more advanced manufacturing solutions. This technology has allowed for product quality, complex machining, high precision, repeatability and productivity. Although the aforementioned areas have stimulated the development of these technologies, their application has been implemented in all areas of human activity. The application of these technologies in the production of orthopaedic aids was first mentioned in the mid-1970s; however, two decades were necessary for them to be intensively applied in this area. Among other factors, this is connected with the development of the digitalization technology, which led to the development of 3D imaging of human body parts of interest [1], and the progress made in the CAD program functionality [2]. Significant problems related to the implementation in the early stages were connected with its practical inconvenience, low production speed and inadequate prices. The majority of such systems was based on the use of functions from the existing CAD program packages that were used by architects and engineers for making technical documentation. Such functionality is adapted to drawing plans for skyscrapers or complex machine parts, but it is complicated to be used for the purpose of correcting a mould model for a prospective aid. Furthermore, engineering tasks typically involve the need for designing a product that is to be manufactured within serial production. Thus, in product development planning a sufficient amount of time is usually reserved for finalizing design details and planning the machine tool trajectories.

Orthoses are aids whose production belongs to the area of the medical-technical profession of orthotics and prosthetics. The design and production of orthoses involves complex geometry of the surface that is to be modified, and this procedure must be carried out quickly. Each product is individual. It is frequently required that an orthosis must be delivered within 24 hours. While the application of CAD/CAM technology in, for example, the automotive industry is currently irreplaceable even with higher initial investments, the orthotic practice for many reasons requires more favourable production solutions. Today there are specialized O\&P CAD/CAM packages available in the market, which represents a significant progress as compared with the situation twenty years ago. Their application has led to improved practical convenience, functions for quick and simple surface manipulation have been added, machine tool trajectories have been generated in an improved manner, and appropriate hardware has been developed in the form of specialized numerical machines. However, the price still represents the limiting factor for wider application. Therefore, numerous companies opt for production at larger laboratories that are adequately equipped, which has become a general trend [3].

Spinal orthoses are used in the treatment of many locomotive system disorders for the purpose of supporting cervical, thoracic and lumbar spine as well as junctional regions [4]. Among frequent conditions in which spinal orthoses are used, the application in scoliosis, ankylosing spondylitis, paraplegia, osteoporosis, conditions following a trauma, surgery, intervertebral disc herniation and/or prolapse, and generally in vertebra fractures regardless of the aetiology are to be emphasized. Orthoses, along with other benefits, allow for spinal stability during walking, standing and lying, control spine posture during movement, redistribute the pressure, provide resistance to external forces, and limit spine movements within the required scope [5]. In the market they are available in special forms as prefabricated products that may partially be adapted to match the desired requirements, or they are made individually. In the conventional procedure the patient is wrapped in plaster bandage while standing upright or in a modified position. As it hardens, the plaster bandage assumes the shape of the body part it was applied to. Such mould is subsequently taken off, closed and filled with plaster or polyurethane mixture.

As the material that the primary mould is filled with hardens, the mould falls off and a solid model of the body part is obtained, which represents the foundation for desired modifications. In the conventional procedure this aspect represents a significant problem because it is timeconsuming, while certain corrections, such as the ones in the case of scoliosis, require significant modifications on the model. The conventional plaster model is rigid and may not be plastically deformed. Therefore, a lot of work has to be invested in order to make an adequate mould out of it, whereupon a special problem arises that is related to maintaining volume relations between the segments. Some 
practices use the base of pre-made plaster casts as the foundation for modifications. For each patient a mould closest to the perimeter and distances on body sections is selected from the warehouse and is manually modified to reach the desired values. The problem in this procedure is that each modification results in alterations of the existing mould, which has to be monitored and recorded.

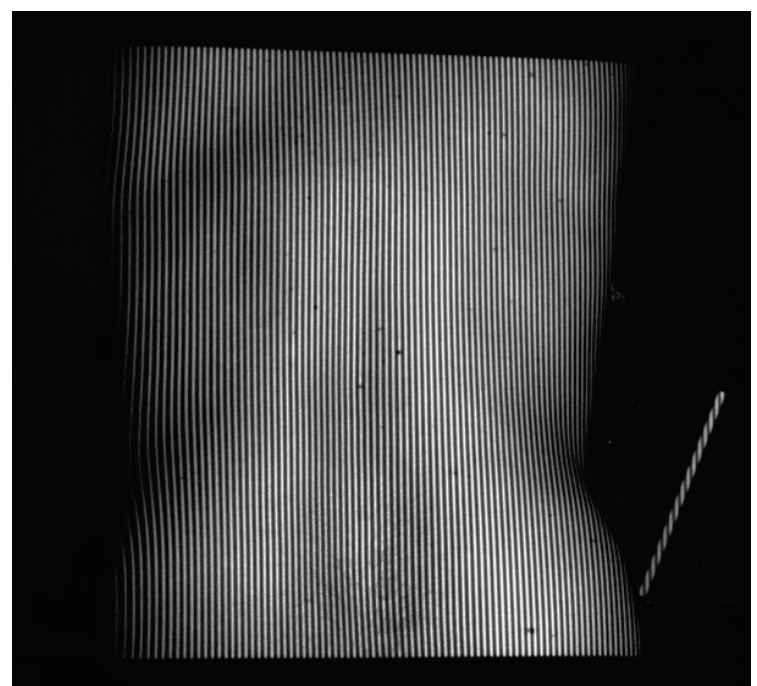

Figure 1 Surface of the back taken by a contactless 3D digitizer

Furthermore, moulds modified in this manner lose quality due to frequent modifications, so they have to be replaced after multiple use. This approach requires a warehouse with a larger number of models for the purpose of selecting the one with the most similar measures and that requires the lowest number of corrections, which additionally contributes to the complexity of the procedure.

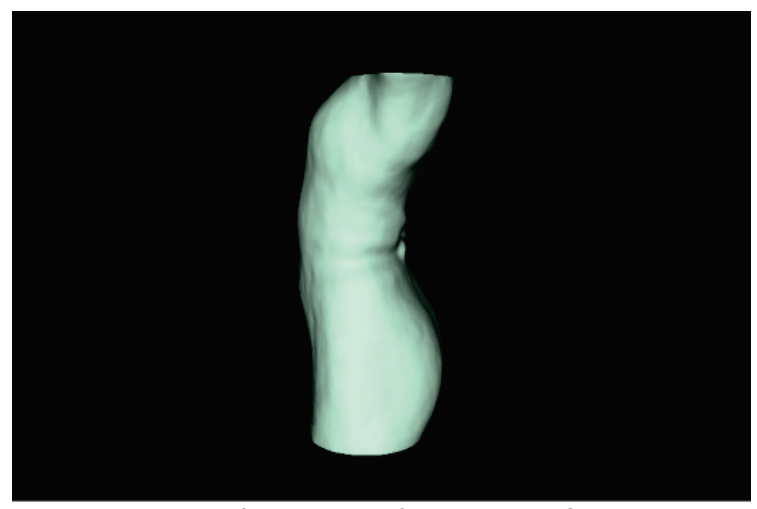

Figure 2 Model of a torso, ready for import into a CAD program

Modern digital technologies have provided numerous answers to questions coming from the practice. They have allowed for body digitalization by means of contactless digitizers, which has significantly simplified the volumetric measurement procedure both for the patient as well as for the professionals conducting the imaging. The results are obtained in a digital form and are processed in advanced CAD programs whose functionality allows for manipulation and obtaining of adequate corrective solutions in the design, which has significantly improved the orthosis functionality.

In addition, design results may be exported and analysed in FEA programs. When the design is completed, the trajectories for a numerical machine tool, a robotic hand or another device that will be used for producing the orthosis mould are generated. Although trends in the selection of a manufacturing technology are rapidly changing and relying on the use of additive technologies, current issues related to this technology are still a limiting factor in its wider application in the production of orthoses. Thereat, the problems of production speed and the question of the mechanical properties of the finished product are particularly emphasized, because these are products that occupy a significant volume, but have thin walls, large surfaces and must be lightweight. In addition, the price is high and the physical size of such systems that would make it possible to produce the whole orthosis is still large. Industrial robotic hands are increasingly used in the production of orthosis moulds [6]. Robotic arms can have special features that provide them with great flexibility compared to other production approaches. However, as a rule they require the use of additional hardware, especially the rotating table. The use of a robotic arm in the production of orthoses is characterized by the ability to make complex mould designs and finished products (e.g. custom-made seats or soft inserts for wheelchairs and chairs).

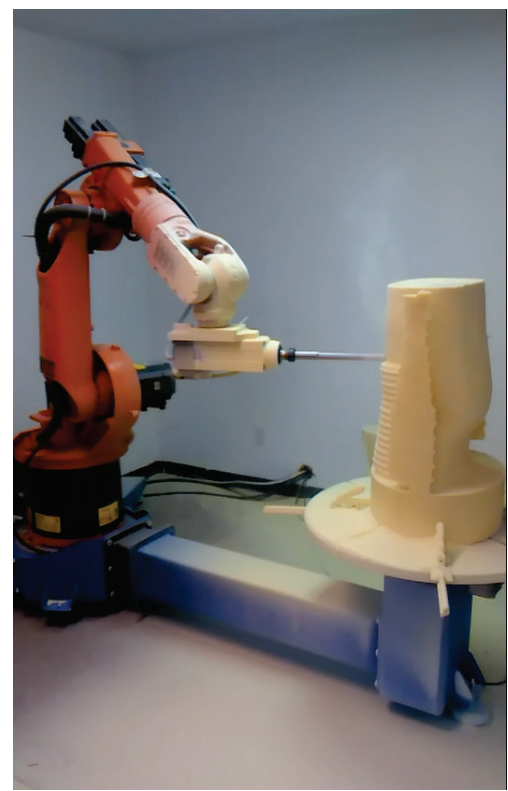

Figure 3 Production of a spinal orthosis mould using an industrial robot

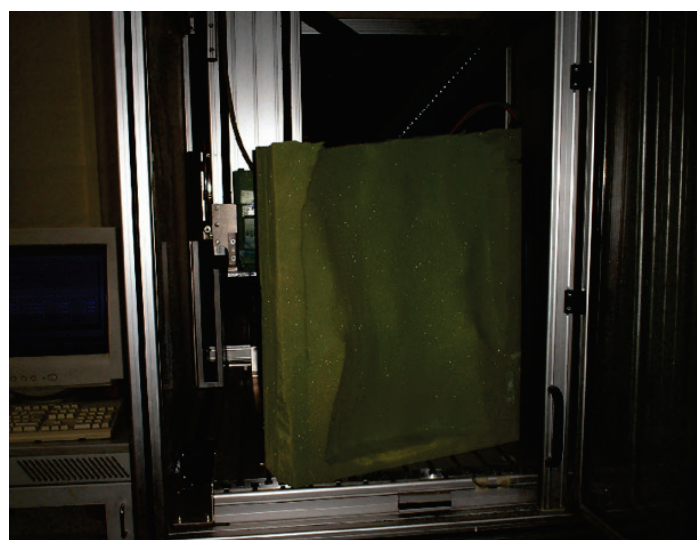

Figure 4 Orthosis made of sponge material using a CNC machine

The negative side of the implementation of this technology also relates to the price of the entire assembly 
and the significant complexity of the strategy of assigning processing trajectories. The latter is especially important to be emphasized, because practical application in the O\&P practice requires this process to be quick and very simple. Certainly the simplest approach to mould production implies the use of special numerical machines [7, 8]. The features and functionality of numerical machines are tailored to the specified requirements. As in the aforementioned cases, the problem here is also related to a high initial investment.

Due to high costs, small practices are reluctant to buy such equipment, as it raises the question of the viability of the investment and the duration of the return (which is often questionable) of the invested financial means. Consequently, the market is expected to respond well to any machine solution that involves appropriate functionality, performance, robustness and an acceptable investment amount. This task is not easy to accomplish. In its implementation a dose of precaution is always required, as the solution to the problem is not primarily related to the implementation of cheap components, but to innovative construction solutions. While considering the existing performance of the machines used for the aforementioned application and generally the needs of small laboratories, several important conditions are emphasized that need to be met in order to ensure that such a machine is functionally suitable and well received. In this case, resources are significantly limited, i.e. a lack of money, people, space and generally technical means is almost always present, which complicates the implementation of new technologies [3].

The machine is required to be:

- of favourable price

- suitably fast and functional

- of small dimensions

- robust

- of adequate ergonomics

- $\quad$ simple for training and use

- simple from the aspect of serving the material and cleaning waste material

- suitable for frequent changes of location within the work space

- constructed in the manner that parts that are frequently replaced or maintained are easy to approach

- silent

- $\quad$ energy-efficient

- adequately safe.

It is evident that the issue is complex, especially from the aspect of requirements related to the low price of the machine. Literature and other sources of information that describe the existing designs are scarce, which may partially be explained by the fact that these are protected commercial solutions. However, there is ground for considering a possible concept whose features could come closer to the required conditions. In the text to follow, the authors consider their proposal of a machine of an unconventional design. A fundamental difference between this proposal and the solutions available in the market is the feature of $Y$ and $C$ axes being coupled and mutually dependent. This allows for the possibility of reducing the number of active components, as well as the possibility of making more complex designs, as discussed in the text to follow.

\section{METHODOLOGY}

The proposal of the machine concept involves significant differences from the aspect of construction as compared with the usual practice. The differences are observable both from the aspect of generating trajectories, as well as from the aspect of the control mode. The concept is based on the reduction of all mechanical components until an acceptable minimum is reached while maintaining the required functionality, which is expected to result in desired economization and making it more competitive as opposed to the existing commercial solutions. The majority of such machines is constructed in three- or four-axes design (rarely five-axes machines may be found too), where the $C$-axis is positioned horizontally or vertically within the coordinate system with a defined relation toward the $X Y Z$ coordinate system of the machine. In the proposed solution (Figs. 4, 5 and 6 ) the $Z$-axis with a spindle motor and a milling cutter moves vertically toward the rotation axis of the $C$-axis, and parallel to it, as it is fixed to the $Y$ axis. The $Y$-axis is parallel to the rotation axis of the rotating table, i.e. the $C$-axis.

The idea of the procedure is as follows:

- The motor that turns the rotating table is coupled with the threaded spindle of the $Y$-axis via a pulley (or a cardan joint)

- The raw part is fixed onto the rotating table and a special program is used for generating tool trajectories for the required kinematics

$$
\varphi_{\mathrm{s}}=\varphi_{\mathrm{p}}
$$

there at the speed ratio is defined as $i_{\mathrm{R}}=$ const. (2)

- $\quad$ The threaded spindle of the $Y$-axis has a defined pitch and its distance from the rotation axis of the rotating table amounts to a defined value. The rotation axis of the rotating table and the axis of the threaded spindle are parallel

$$
P_{\mathrm{h}}=\text { const. }
$$

- As it is known which table turn is required for the pitch value on the $Y$-axis

$\varphi_{\mathrm{s}}=2 \pi n_{\mathrm{s}}$,
$h_{\mathrm{vr}}=n_{\mathrm{vr}} P_{\mathrm{h}}$.

- The total number of possible rotations of the $Y$-axis is defined by the effective length of the $Y$-axis divided by the pitch of the threaded spindle. If one knows the pitch of the threaded spindle and the speed ratio as related to the rotating table, one may easily determine for which amount the $Y$-axis is going to turn, according to the given turn of the rotating table

$$
\begin{aligned}
& h_{\mathrm{vr}}=n_{\mathrm{vr}} P_{\mathrm{h}}, \\
& h_{\mathrm{vr}}=\frac{\varphi_{\mathrm{s}}}{2 \pi i_{\mathrm{R}}} P_{\mathrm{h}},
\end{aligned}
$$


$h_{\mathrm{vr}} \| Y$ axis,

- The proposed design enables a joint drive and the dependence between the motion of the rotating table and the threaded spindle, i.e. the $Y$-axis and the axis

$\left[\left(\varphi_{\mathrm{vr}}\right)\right]\left(=f\left(\varphi_{\mathrm{s}}, i_{\mathrm{R}}\right)\right)$,

The $Z$-axis is fixed vertically onto the $Y$-axis $C \perp Z,(10)$

- The spindle motor with a milling cutter is positioned on the $Z$-axis. The motion direction of the milling cutter is along the line vertical to the $C$-axis - the rotation axis of the rotating table

\section{$Y \perp Z$.}

- The motor that drives the $Z$-axis is independent of the motor that drives the $C$-axis (rotating table) and the $Y$ axis.

The raw part is fixed onto the $\mathrm{C}$-axis acceptance. For the purpose of making a spinal orthosis mould, it is mostly made of hard, expanded polyurethane shaped in the cylindrical form with a shaft or fixation slots. It must be emphasized that the application of such material for making moulds represents a great advantage in comparison to the application of plaster in the conventional procedure, as it reduces the mould weight by 5 to 10 times, so the mould may be easily worked with. Furthermore, this material is much softer and more convenient for additional processing. A more complex, 4-axes design of the machine involves a work space in which the $\mathrm{Z}$-axis moves on the portal in the XY plane and treats the raw part that is fixed on the $\mathrm{C}$-axis. This allows for a higher quality level of treatment, as it reduces the possibility for surface parts to be inaccessible for the tool. In addition, the quality of the treated surface is significantly better, as the milling cutter, at least from one direction, is positioned more conveniently towards it, but the implementation of the $X$-axis is required. However, in the spinal orthosis production, although better quality of the treated surface is always welcome, the crucial issue is connected to achieving an optimum relation between its roughness and processing speed, which is due to the fact that greater production speed is required. Therefore, milling cutters of larger diameter are used, and greater feed rates during the processing are required. In the end it is possible to smooth the mould surface up to an acceptable level by means of a very simple and quick manual treatment. This fact is useful in considering an alternative approach, as it makes it possible to determine a fixed pitch for a certain machine when the trajectory is determined along a defined curve; a spiral in this case. The importance of the aforementioned fact relates to the possibility of making the $Y$-axis rotation dependent of the $C$-axis rotation by means of coupling over a belt transmission or geared cardan, with a defined speed ratio. This allows for the reduction of the number of active components by one, i.e. the motor that turns the $C$-axis is used for turning the $Y$-axis as well by means of suitable transmission.

In this simpler design where the $Z$-axis with the spindle motor and the milling cutter that moves vertically to the direction of the $C$-axis, is positioned vertically to the Yaxis, it is possible to make a machine that allows for processing along the planned spiral trajectory. For each turn unit of the $C$-axis the pitch of the $Y$-axis is known, and at the reached height/point the $Z$-axis moves to the defined position from the rotation axis of the $C$-axis and treats the raw part along the way.

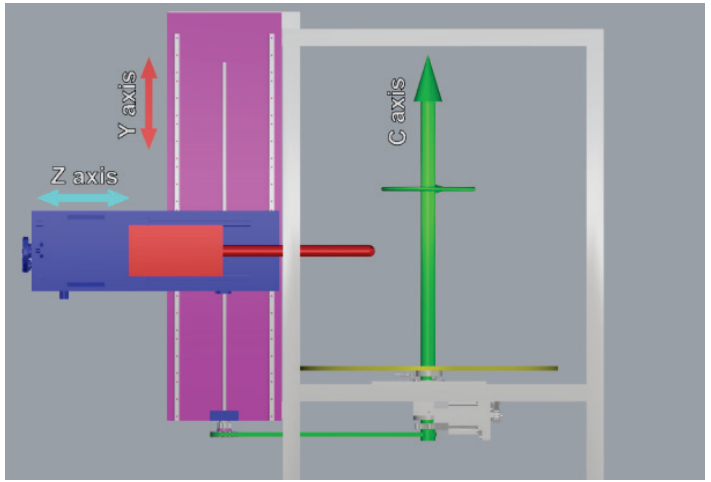

Figure $\mathbf{5}$ Schematic of the model of the proposed machine with the belonging axes

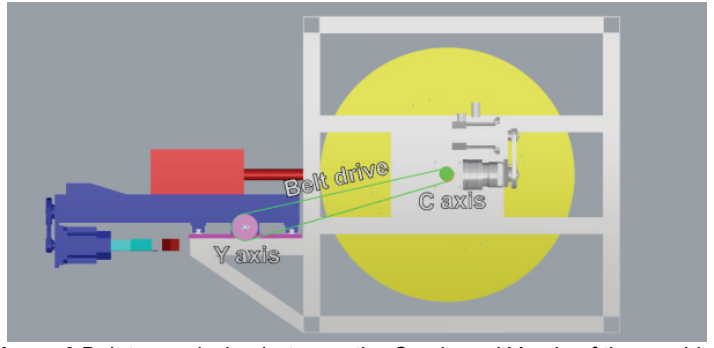

Figure 6 Belt transmission between the $\mathrm{C}$-axis and $\mathrm{Y}$-axis of the machine

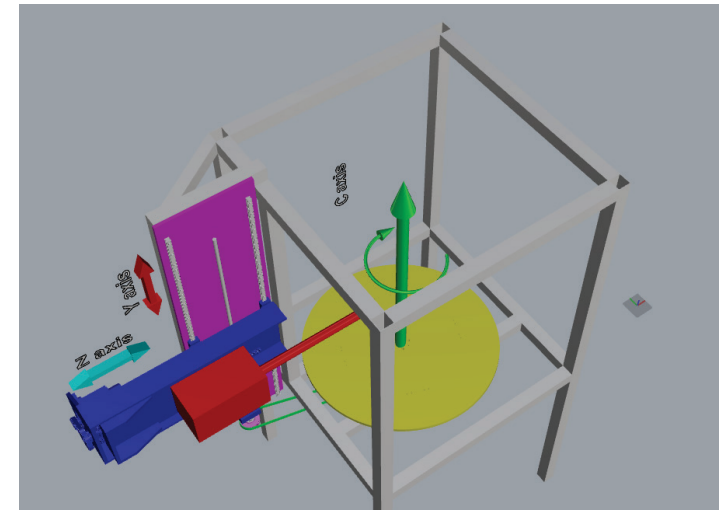

Figure 7 Perspective representation of the machine model and the relation between the axes. The red colour is used for representing the model of the spindle motor with a milling cutter

For this purpose, a special processing simulation software is being developed. Its functions will allow for setting up a virtual torso model in the machine's work space. This space is located within the defined dimensions above the rotating table onto which a raw part with dimensions suitable for the model volume is fixed. Owing to a simplified 3-axes machine design, the software will allow for the model to be turned within the work space, as well as for the dynamic representation of zones (e.g. by colouring these parts differently) that will be unavailable for treatment. In this manner the user may manually reduce them to a significant extent by turning the model. It may also be taken into account that in one of the future designs the possibility of developing an automated process is 
considered. While performing this procedure, the mass displacement of the raw part is to be considered as well in order to reduce vibrations and maintain the stability during processing.

A special software module will be in charge for the issue of generating the tool trajectories. The parameters in this case involve the known distance from 0 point of the $Z$ axis to the rotation axis of the $C$-axis, and the length of the spindle motor with the belonging milling cutter from the referent point on the $Z$-axis. The known parameters also include the milling cutter radius and the diameter of the cylindrical raw material.

The task of this software module is to determine the positions of each point being treated, to define suitable parameters for it, and to store it in a suitable program structure. Upon model orientation in the virtual work space of the machine, the tool trajectories are generated. In the simulation the model fixed to the machine is turned by the known angle amount. As the speed ratio is known, the $Y$ axis with the defined pitch is turned as well. The transversely fixed $Z$-axis is lifted along it.

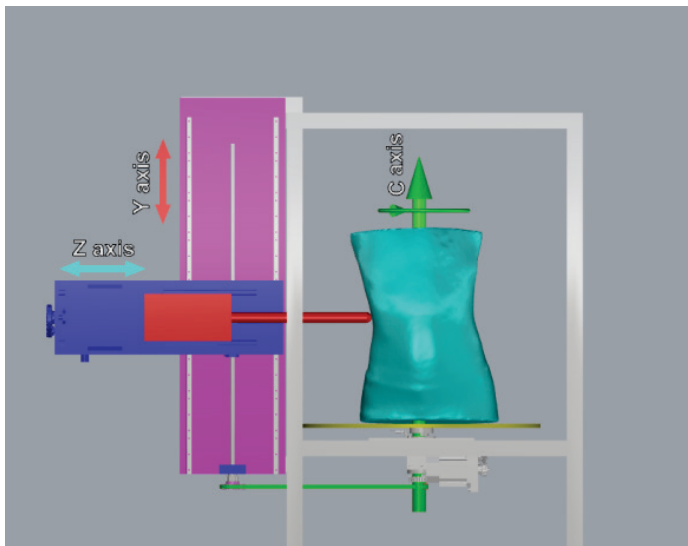

Figure 8 Model of the spinal orthosis mould placed on the rotating table of the machine in the virtual interface of the tool trajectory generating software.

The program measures the length of the line segment that passes through the milling cutter axis from its intersection point with the model surface (closest to the $Y$ axis) to the point on the same line that is closest to the $Y$ axis. This length is reduced by the length from the top of the milling cutter to the point in which the spindle motor is connected to the linear guide of the $Z$-axis. The obtained difference represents the stroke length of the $Z$-axis for a targeted point on the surface. This procedure involves additional procedures, such as compensating for the tool trajectory by the milling cutter radius (it is assumed that the surface is treated using a tool with a rounded top).This procedure is carried out until the trajectory for the whole model is completed and visualized as a series of points in the form of a curve that is equidistantly formed around the model surface, where each point shows the position of the top centre (sphere) of the milling cutter in relation to the corresponding surface. The aforementioned software module that generates the tool trajectories is in the realization process at the moment of writing this paper.

\section{DISCUSSION}

This review considers the concept of a machine whose features might contribute to a more efficient implementation of $\mathrm{CAD} / \mathrm{CAM}$ technology in the production of spinal orthoses at small orthotic laboratories. Although several solutions are available in the market, whose functionality meets the needs of the production of spinal orthosis moulds, there is still a need for a more economical production technology. The crucial issue involves the price, but there are other criteria as well (as mentioned in the text) that should, at least partially, be met in order for the implementation to pay off in the end. The possibility of reducing the number of active components (if the conditions allow for it) may contribute to a lower price of the machine to a considerable extent. This approach considers the possibility of connecting the movements of the $C$-axis and $Y$-axis while implementing a suitable speed ratio and creating a defined trajectory along which the $Y$ axis pitch is known for each rotation amount of the table that the raw part is fixed to. The speed ratio may be adjusted to meet specific needs and in this manner the required pitch and the overlap density for a certain milling cutter type may be achieved.

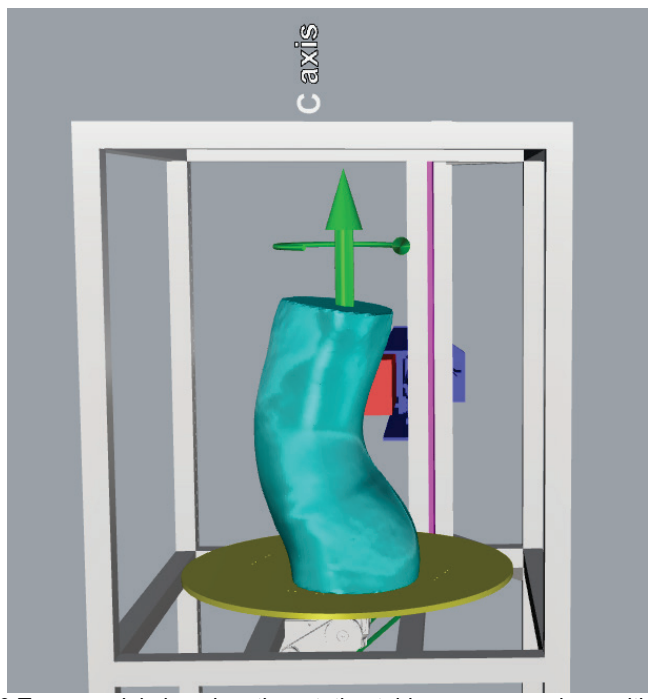

Figure 9 Torso model placed on the rotating table upon manual repositioning for achieving a minimum amount of hidden surface during processing.

The fact remains that the production of spinal orthosis moulds is not especially demanding from the aspect of details, which simplifies further work for the purpose of defining the concept based on the presented model. Furthermore, there is no significant need for frequent changes of the overlap density of the milling cutter along the surface, as surface accuracy in the sense of a physical reproduction of a CAD model is not a primary requirement, but the surface, along with following the CAD model shape, is expected to be smooth enough in order for additional processing to be simplified (this relates to manual smoothing of the surface - the procedure is very short, especially on models made of expanded polyurethane). Regarding the issue of machine control, standard control cards mostly have outputs for four motors. This allows for the theoretical possibility to use a single card for controlling two machines of this type. In addition, it is theoretically possible (as the $Y$-axis and the $C$-axis are coupled) to use the rotation of one $C$-axis for driving several equal configurations via another transmission mechanism. For example, it is possible to drive two machines with three motors. 


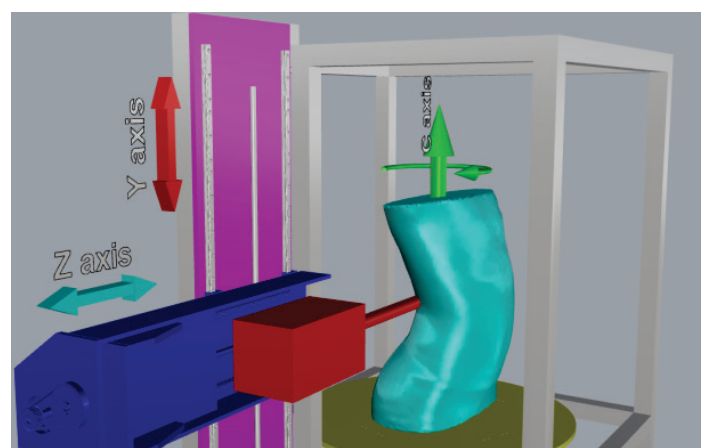

Figure 10 Representation of the production procedure of a spinal orthosis mould in the simulation software

Although these are theoretical assumptions, the authors' previous work points to the likelihood of realizing such a simplified machine and of making it functional in practice. A contributing factor is the fact that softer materials are used in the production of spinal orthosis moulds, such as expanded polystyrene or expanded polyurethane, where treatment forces are not great.

\section{CONCLUSION}

This unconventional concept of a machine design is new and different from other solutions available in the market. It is suitable for a simpler, quicker and cheaper implementation of CAD/CAM systems at smaller orthotic practices. The activities carried out so far point to the possibility of realizing such a machine from the aspect of construction and kinematics.

Future work involves the development of the mathematical model for generating tool trajectories as related to the kinematic model, as well as the construction of a simulator that should show the functionality of the machine in the virtual interface. Should the outcome of the aforementioned activities be positive and yield satisfactory results, it will be a foundation for initiating the realization of a physical concept.

\section{REFERENCES}

[1] Phillips, O. J. (2008.). New Applications of CAD/CAM, The O\&P EDGE. Retrieved from https://opedge.com/Articles/ ViewArticle/2008-09 01

[2] Kennedy, S. (2009.). The Future of O\&P: A Practitioner's Perspective, The O\&P EDGE. Retrieved from https://opedge.com/Articles/ViewArticle/2009-01-01/20090104

[3] Smith, D. G. \& Burgess, E. M. (2001). The use of CAD/CAM technology in prosthetics and orthotics Current clinical model sand a view to the future. Journal of Rehabilitation Research and Development, 38(3), 327-334.

[4] Agabegi, S. S., Asghar, F. A., \& Herkowitz, H. N. (2010). Spinal orthoses. J Am AcadOrthop Surg., 18(11), 657-667. https://doi.org/10.5435/00124635-201011000-00003

[5] See http://www.albertaoandp.com/spinal-orthoses

[6] See http://rodin4d.com/en/Products/manufacturing/victorrobot

[7] See http://vorum.com/cad-cam-prosthetic-orthotic/carvers/

[8] See http://rodin4d.com/en/Products/manufacturing

\section{Contact information:}

Karlo OBROVAC, MD

(Corresponding author)

ORTOGEN d.0.0.

Remetinec 42, 10000 Zagreb, Croatia

karlo.obrovac@zg.htnet.hr

Pero RAOS, PhD, Full Professor

Mechanical Engineering Faculty in Slavonski Brod

Trg I. B. Mazuranic 2, 35000 Slavonski Brod, Croatia

praos@sfsb.hr

Tomislav GALETA, PhD, Associate Professor

Mechanical Engineering Faculty in SlavonskiBrod

$\operatorname{Trg}$ I. B. Mazuranic 2, Slavonski Brod, Croatia

tgaleta@sfsb.hr

Josip NIŽETIĆ, graduate engineer

COGNITUS d.0.0.

Remetinec 42, 10000 Zagreb, Croatia

josip.nizetic@cognitus.hr

Alan MUTKA, PhD

Bjelovar University of Applied Sciences

Trg Eugena Kvaternika 4, 43000 Bjelovar, Croatia

amutka@vub.hr

Jadranka VUKOVIĆ OBROVAC, MD

ORTOGEN d.0.0.

Remetinec 42, 10000 Zagreb, Croatia

j.v.obrovac@gmail.com

Zoran VRHOVSKI, MSc

Bjelovar University of Applied Sciences

Trg Eugena Kvaternika 4, 43000 Bjelovar, Croatia

zvrhovski@vub.hr

\section{APPENDIX}

O\&P - Orthotics \& Prosthetics

CNC - Computer Numeric Control

CAD - Computer Aided Design

CAM - Computer Aided Manufacturing

FEA - Finite Element Analysis

$\varphi_{\mathrm{s}} \quad$ - turn angle of the table, rad

$\varphi_{\mathrm{p}} \quad$ - turn angle of the raw part, rad

$i_{\mathrm{R}} \quad$ - speed ratio of the belt transmission

$n_{\mathrm{s}} \quad$ - number of table rotations

$n_{\mathrm{vr}} \quad$ - number of spindle rotations

$h_{\mathrm{vr}} \quad$ - height of lifting the nut with the spindle milling cutter along the $Y$-axis, mm

$P_{\mathrm{h}} \quad$ - threaded spindle pitch, $\mathrm{mm}$

$X, Y, Z$ - axes in the Cartesian coordinate system 